\title{
Unveiling unresolved stellar components from photometry
}

\section{The case of TWA 23 and the importance of pre-whitening}

\author{
Sergio Messina ${ }^{1}$ (D) Berto Monard $^{2}$
}

Received: 8 April 2021 / Accepted: 18 January 2022 / Published online: 28 January 2022

(c) The Author(s) 2022

\begin{abstract}
Space-born missions designed to search for exo-planets are providing us with highprecision photometric time series very well suited to measure the rotation period of solar- and lower-mass stars. Considering the large number of stars monitored during the mission life, the possibility to inspect each periodogram is out of question and, therefore, each method to search for rotation periods applied either individually or in combination is designed to automatically select the best rotation period estimate. In the case of unresolved binary stars it is possible to measure the rotation period of both components, when these are both variable and have comparable magnitude. On the other hand, the detection of two different rotation periods from the same photometric timeseries can represents a tool to unveil the binary nature of the star. In this paper, we present the case of the star TWA 23, a member of the young TWA association, as just one example of numerous cases. These may occur when the star is an unresolved binary and the variability of the secondary component is present in the time series. However, the frequency of the primary and dominant power peak in the periodogram, originating from the primary component, needs to be pre-whitened to allow the frequency of the secondary component to be detected. Considering the possible presence of undiscovered close binaries in the input catalogs of various ongoing or future missions aimed at exoplanet search, it becomes advisable to follow routinely this filtering approach to prevent to miss relevant information on the true binary nature of stars.
\end{abstract}

Keywords Stars: low-mass $\cdot$ Stars: rotation $\cdot$ Stars: activity $\cdot$ Stars: pre-main sequence $\cdot$ Individuals: TWA 23

Sergio Messina

sergio.messina@inaf.it

1 INAF- Catania Astrophysical Observatory, via S. Sofia 78, I-95123 Catania, Italy

2 CBA-Kleinkaroo, Klein Karoo Observatory, PO Box 281, 6660 Calitzdorp, South Africa 


\section{Introduction}

Rotation is a key stellar parameter in the physics of low-mass stars. On the one hand it drives the dynamo generation of magnetic fields (e.g., [13, 27]). On the other hand, magnetic fields control the evolution in time of stellar rotation, either by a redistribution of internal angular momentum, through the coupling or decoupling of internal radiative core with the external convective envelope [23], or by the angular momentum loss, through the braking mechanisms by magnetized stellar winds [21]. The time dependence of stellar rotation is exploited to estimate the stellar age by means of the gyrochronology [3], that is by means of empirical relations between mass, rotation, and age which are calibrated using benchmark stellar open clusters.

The knowledge of stellar rotation is relevant in exo-planetary studies. In fact, the rotation period of the host star allows to constrain its age and, consequently physical and orbital properties of their planetary companions and to understand the evolution of planetary systems. As well, the knowledge of the rotation period allows to disentangle radial velocity variations arising from magnetic activity from those of Keplerian nature induced by the planetary companion (e.g., [1]).

Both ground-based projects and space-born missions aimed at the discovery of exoplanets by means of the transit method provide time series of stellar flux measurements which are well suited to infer the stellar rotation period. Among the period search methods generally used, we just recall the Generalized LombScargle (GLS; [28]), which is well suited also for unevenly sampled time series, and the Autocorrelation Function (ACF; [4]). The first operates in the frequency domain whereas the latter in the time domain. Other methods, which are found with lower frequency in research papers are the CLEAN [18], the Wavelet analysis [26] and the Phase Dispersion Minimization [24].

The most robust determination of the rotation period generally comes when two or more methods are applied either individually, with a posteriori comparison of their results, or in novel combination (see, e.g. [19]). The periodogram analysis of Kepler data has revealed a significant fraction of low-mass stars with multiperiodic flux variations (see, e.g., [17]). Most of these multiperiodic stars turned out to be unresolved close binaries [11, 25]. Indeed, high-precision longtime series photometry revealed to be a powerful tool to detect and unveil the nature of unresolved close binaries.

In the present paper, we present, as an example case, the close binary TWA 23 to show which would be the most advisable approach in the rotation period determination. In fact, a straitforward application of both GLS and ACF on the high-quality TESS photometry fails in detecting the presence of a secondary periodicity arising from the spatially unresolved secondary component. Whereas even a visual inspection allows to measure the stellar rotation period of the primary component, nonetheless the secondary periodicity, although present with high significance in the time series, remains hidden from the ACF and GLS methods, unless the primary period is pre-whithened. Differently, the CLEAN method, where the filtering process is integral part of the method, is capable to detect the 
secondary periodicity without an additional filtering. Very briefly, CLEAN first computes the power spectrum, as GLS does, and the spectral window function whose power peaks depend on the data sampling and are responsible for the aliasing. Then, CLEAN iteratively centers the window function at the frequency of the highest periodogram power peak (the one expected to be of astrophysical origin) and removes a fraction of its power from it (the highest peak and its possible alias are then reduced in power). Contemporary, that fraction of power removed from the highest peak is transferred to the same frequency in the clean spectrum. This procedure iteratively removes the highest power peaks (in our case the peaks of the primary and the hidden peak of the secondary component) and its alias features introduced by the windows function and build up a cleaned spectrum with the only frequencies related to the astrophysical signals.

In our opinion, the pre-whitening of the dominant periodicity should be routinely applied in the pipelines of reduction and rotation period determination of the recently completed (like Kepler), ongoing (like TESS) and future (like PLATO) space missions when either GLS or ACF are used.

\section{Literature information}

TWA $23\left(\mathrm{RA}_{\mathrm{J} 2000}=12: 07: 27.44 ; \mathrm{DEC}_{\mathrm{J} 2000}=-32: 47: 00.0 ; \mathrm{V}=12.55 \mathrm{mag}\right)$ is a southern SB spectroscopic binary consisting of M-type components.

Shkolnik et al. [20] first detected spectroscopically both components allowing to classify this star as a SB2. In their study, they assigned the same M3 spectral type to both components. Subsequent analysis by [2], based on time series spectra, revealed a long-timescale $(>1 \mathrm{yr})$ trend in the radial velocity $(\mathrm{RV})$, confirming the binary nature. The small semi-amplitude $\left(\sim 5 \mathrm{~km} \mathrm{~s}^{-1}\right)$ of the RV variations suggested that the secondary component may be a lower- rather than an equal-mass star as assumed by [20]. Although the orbit is still undetermined, they could establish an upper limit on its orbital period. They find three plausible orbital solutions with orbital periods of 517,777 , and 1552 days. The binarity of TWA 23 was recently confirmed by [7] who detected clear evidence of a double peak in the Cross Correlation Function of a FEROS (Fiber-fed Extended Range Optical Spectrograph) spectrum, revealing the binary nature of this target. However, owing to an uncertainty (of $1^{\prime}$ ) in the coordinates of this spectrum with respect to previous observations, they did not give much trust that the spectrum was actually of this target. [29] added one more UVES (Ultraviolet and Visual Echelle Spectrograph) spectrum to literature RV data and confirmed the RV variation noted in previous studies and the significant asymmetry in the line profile causing a large bisector slope, suggesting this stars is indeed a SB2 system.

Song et al. [22] first suggested that TWA 23 could be potential member of the young 8-Myr southern TWA association. However, they had no complete kinematic data to test its membership with the convergence method. This membership is now confirmed with $99.2 \%$ probability by the BANYAN $\Sigma$ tool (Bayesian Analysis for Nearby Young AssociatioNs $\Sigma$ ) by [8] taking advantage of recent kinematic and distance data made available by GAIA. The young age of TWA 23 is supported by 
evidences of relevant magnetic activity at all atmospheric levels. [22] measured the chromospheric $\mathrm{H} \alpha$ line in emission $\left(\mathrm{EW}_{H \alpha}=-2.2 \AA\right)$. [9] report similar emission level $\left(\mathrm{EW}_{H \alpha}=-2.44 \AA\right.$ ). [5] report $\log \mathrm{L}_{\mathrm{X}}=29.17 \mathrm{erg} \mathrm{s}^{-1}$ and $\log \mathrm{L}_{\mathrm{X}} / \mathrm{L}_{\mathrm{bol}}=-2.94$ for the X-ray luminosity derived from ROSAT observations. The Li EW measured by [22] $\left(\mathrm{EW}_{L i}=460 \mathrm{m \AA}\right)$ and by [10] $\left(\mathrm{EW}_{\mathrm{Li}}=525 \mathrm{m \AA}\right)$ are consistent with the youth of this system.

Two measurements of projected rotational velocity are available in the literature: $v \sin i=14.78 \mathrm{~km} \mathrm{~s}^{-1}$ obtained by [9] with a resolving power $\mathrm{R}=60000$ and a larger value $v \sin i=20.5 \pm 5.5 \mathrm{~km} \mathrm{~s}^{-1}$ by [2], but obtained with a lower resolving power $\mathrm{R}$ $=30000$.

\section{Photometry}

Triggered by the suspicion raised by [20] on the binary nature of TWA 23, we searched for the photometric time series available at that time in the public archives to get an independent confirmation of its binarity, that is aiming at detecting hints of variability from the secondary spatially-unresolved component, if any.

\subsection{Archival photometry}

Three photometric surveys ASAS (All Sky Automated Survey; [15]), Super-WASP (Super Wide Angle Search for Planets; [16]), and CSS (Catalina Sky Survey; [6]) monitored TWA 23 for time intervals of different lengths, with different samplings and photometric accuracies. We retrieved from the respective public archives three magnitude time series that were analysed in similar manner. First, we discarded poor quality measurements (all measurements with an uncertainty 2 times larger than the average), then removed the outliers applying a $3 \sigma$ threshold. Finally, we analysed the time series with the Generalized Lomb-Scargle (GLS) and CLEAN periodograms. Owing to the irregular data sampling, the use of ACF turned out not suited for these datasets.

\subsubsection{ASAS}

TWA 23 is designated as ASAS 120727-3247.0 in the ASAS archive [15]. We retrieved a long data time series consisting of 9 observation seasons lasting from six to nine month each, from Nov 2000 to Aug 2009 for a total of 538 good quality measurements with about 60 measurements on average per season (see Table 1). The ASAS photometry generally consists of one magnitude measurement every one or two days, with an integration time of three minutes in the $\mathrm{V}$ filter and, in the specific case of TWA 23 with an average accuracy $\sigma=0.029 \mathrm{mag}$, whereas the magnitude standard deviation over the complete series is rms $=0.086 \mathrm{mag}$. The ASAS data allowed us to determine the star's brightest level at V $=12.55 \mathrm{mag}$. 
Table 1 Log of observations

\begin{tabular}{llllll}
\hline Survey & $\begin{array}{l}\text { Time range } \\
(\mathrm{yr})\end{array}$ & $\begin{array}{l}\text { \# of meas. } \\
\text {. }\end{array}$ & S/N & Band/Filter & $\begin{array}{l}\text { exp.time } \\
(\mathrm{s})\end{array}$ \\
\hline ASAS & $2000-2009$ & 538 & 35 & V-standard & 180 \\
SuperWASP & $2006-2008$ & 9553 & 25 & $400-700 \mathrm{~nm}$ & 30 \\
Catalina & $2005-2012$ & 276 & 25 & unfiltered & 30 \\
KKO & 2014 & 9581 & 150 & R-standard & 30 \\
TESS & 2019 & 15392 & 1000 & $600-1000$ & 2 \\
\hline
\end{tabular}
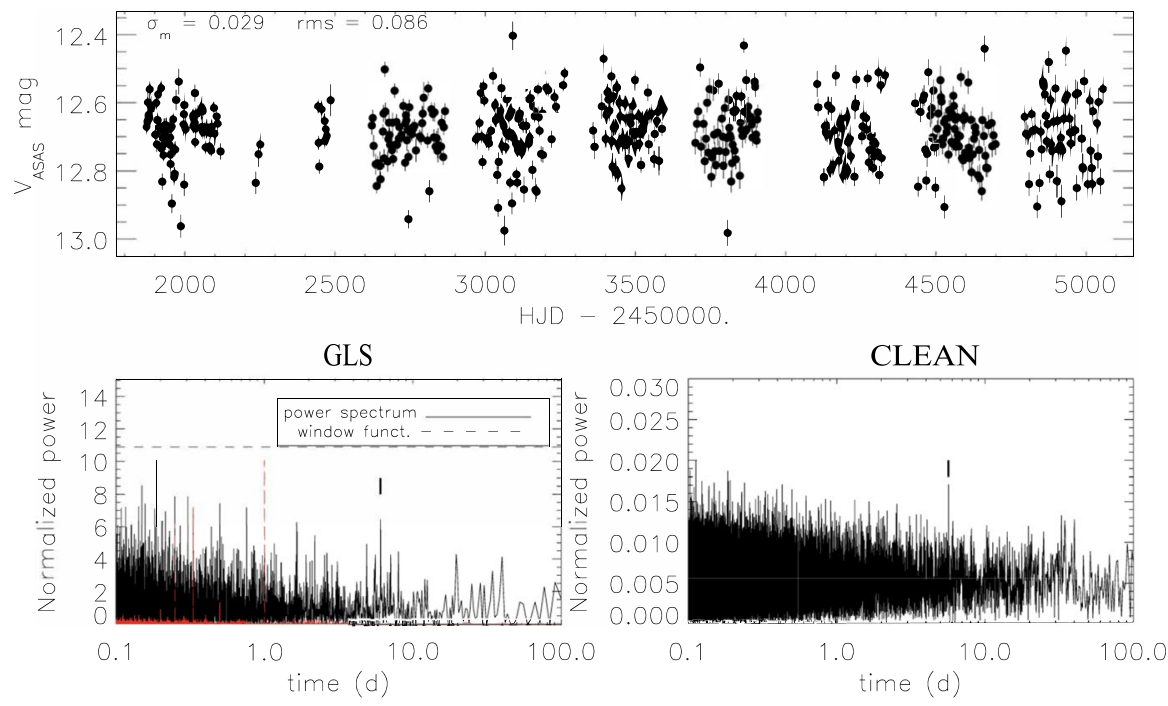

Fig. 1 Summary of periodogram analysis of the ASAS photometry of TWA 23. Top panel: V-band magnitude time series. Bottom left panel: GLS periodogram with indication (dashed line) of the power level corresponding to $99 \%$ confidence level and of the power peak at $88 \%$ confidence level. Bottom right panel: CLEAN periodogram

Our analysis using both GLS and CLEAN periodograms did not reveal any significant power peak at $99 \%$ confidence level. Nonetheless, we noted a power peak at about $\mathrm{P}=5.6 \mathrm{~d}$ in both periodograms at $88 \%$ confidence level.

In Fig. 1 we report a summary of our ASAS data analysis.

\subsubsection{SuperWASP}

TWA 23 is designated as 1SWASP J120733.91-324652.8 in the SuperWASP public archive [16]. We retrieved a data timeseries consisting of 3 observation seasons lasting from three to five month each, from May 2006 to May 2008 for a total of 9553 good quality measurements with about 3100 measurements on average per season (see Table 1). SuperWASP observed TWA 23 generally for 8 consecutive hours per night 

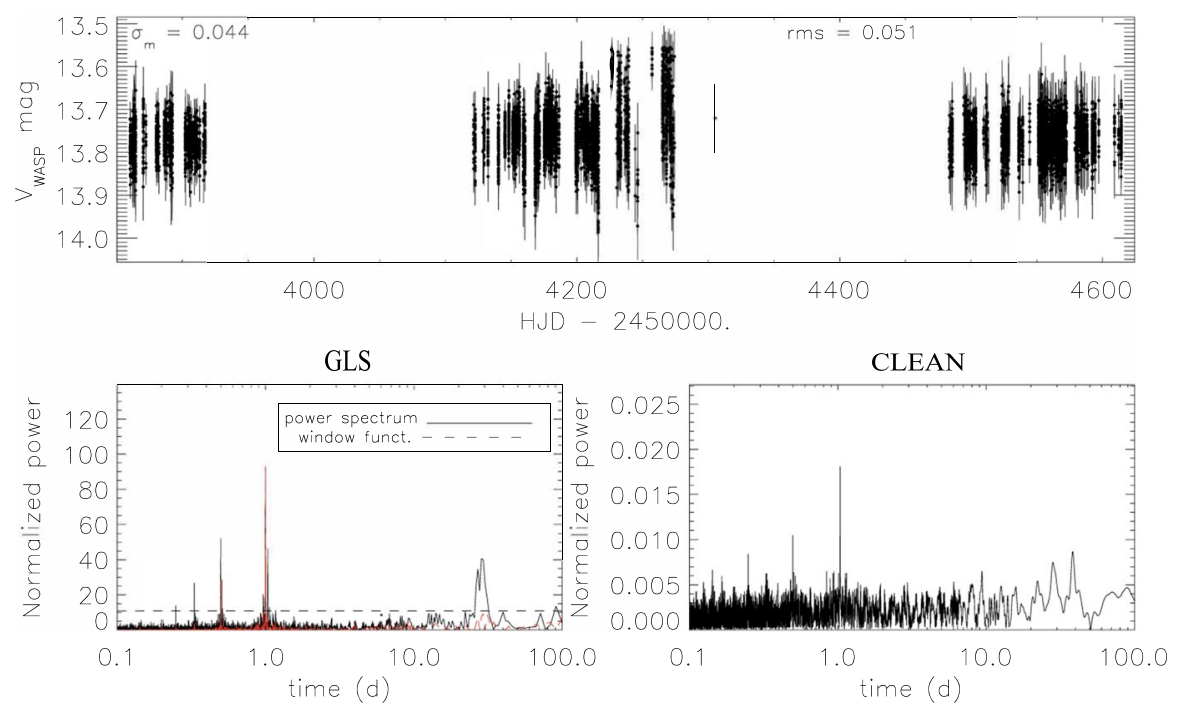

Fig. 2 Summary of periodogram analysis of the SuperWASP photometry of TWA 23. Top panel: V-band magnitude timeseries. Bottom left panel: GLS periodogram with indication (dashed line) of the power level corresponding to $99 \%$ confidence level. Bottom right panel: CLEAN periodogram

with an integration time of 30 seconds per frame and achieving an average data precision is $\sigma=0.043 \mathrm{mag}$. The latter is comparable with the observed amplitude of variability. However, in the second season, we note that the level of variability is significantly higher than in the other seasons, reaching an amplitude of about 0.15 mag.

Our analysis using both GLS and CLEAN periodograms revealed three highlysignificant periodicities: the dominant at $\mathrm{P}=1.00 \mathrm{~d}$, the secondary at $\mathrm{P}=1.033 \mathrm{~d}$, and the third at about 30d. The first and last periodicities arise from the 1-day data sampling imposed by the fixed longitude of observation and the Earth rotation. The CLEAN algorithm, which effectively takes into account the aliasing arising from the spectral window, removed the 1-day and 30-day periodicities, leaving the 1.033d period. [12] suggested this short period to be possibly the stellar rotation period but to be confirmed by additional observations. However, from the present analysis, this turned out to be a spurious periodicity. In Fig. 2 we report a summary of our SuperWASP data analysis.

\subsubsection{Catalina}

TWA 23 is designated as SSS_J120727.3-324700 in the Catalina survey [6]. Catalina observations generally consists of four 30-sec exposures per night and achieved in the case of TWA 23 an average data precision is $\sigma=0.040$ mag (see Table 1). We retrieved a data timeseries from Jul 2005 to Jul 2012 for a total of 276 good quality measurements. Our analysis using GLS periodogram reveals several power peaks at 

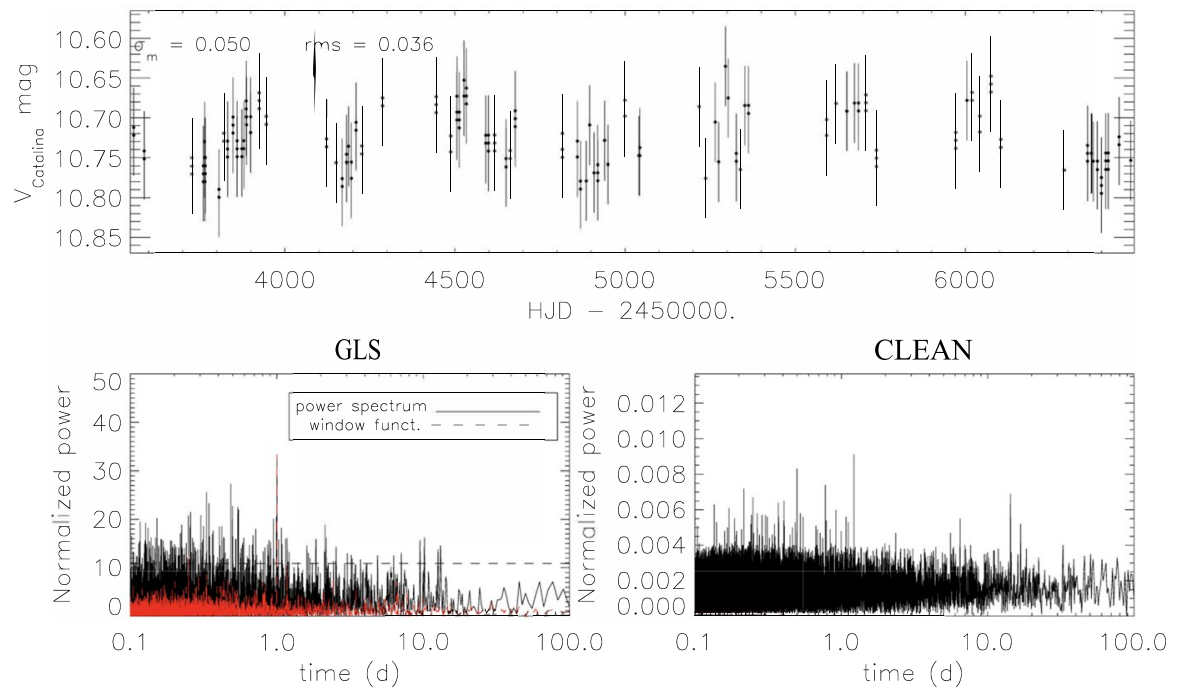

Fig. 3 Summary of periodogram analysis of the Catalina photometry of TWA 23. Top panel: V-band magnitude time series. Bottom left panel: GLS periodogram with indication (dashed line) of the power level corresponding to $99 \%$ confidence level. Bottom right panel: CLEAN periodogram

99\% confidence level. The CLEAN periodogram shows the most significant peak at 1.2d. In Fig. 3 we report a summary of our CSS data analysis ${ }^{1}$.

\subsection{New photometry}

Since our analysis of archival data did not give convincing results, apart from some marginal evidence of a rotation period at $\mathrm{P}=5.6 \mathrm{~d}$, we planned our own photometric monitoring to get higher-quality data with a shorter cadence.

TWA 23 was observed during 24 nights from April 12 to June 11, 2014 at the Klein Karoo Observatory (225 m a.s.l., Western Cape, South Africa). It was observed with a $35 \mathrm{~cm}(\mathrm{f} / 8) \mathrm{RCX}-400$ telescope with a $21^{\prime} \times 14^{\prime}$ field of view, a plate scale of $0.82^{\prime \prime} / \mathrm{px}$, and equipped with the SBIG ST8-XME CCD camera and BV(RI) filter set. We collected a total of 9581 frames in the R filter using a $2 \times 2$ CCD binning. The data reduction was performed using the IRAF $^{2}$ tasks within DAOPHOT. Aperture photometry was used to extract the magnitude series for all stars detected

\footnotetext{
${ }^{1}$ From the archive we report that CSS images are all taken unfiltered. Magnitudes are provided in V-band after a rough transformation and photometric calibration is based on a few tens of stars within each frame.

${ }^{2}$ IRAF is distributed by the National Optical Astronomy Observatory, which is operated by the Association of the Universities for Research in Astronomy, inc. (AURA) under cooperative agreement with the National Science Foundation.
} 

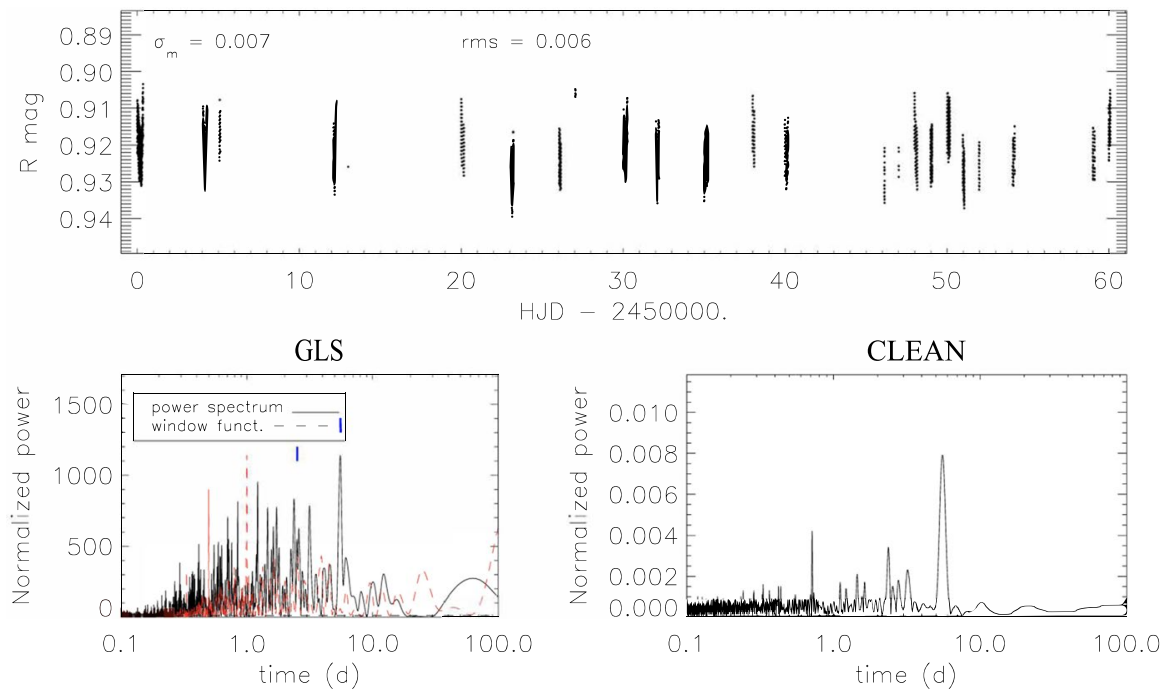

Fig. 4 Summary of periodogram analysis of the KKO photometry of TWA 23. Top panel: R-band magnitude timeseries. Bottom left panel: GLS periodogram with indication (dashed line) of the power level corresponding to $99 \%$ confidence level. Bottom right panel: CLEAN periodogram

in the frames. For each night, the observations were performed for up to 8 consecutive hours.

After bias subtraction and flat fielding, we extracted time series photometry for each detected star. The aperture radius on each night was selected according to the variable seeing and sky transparency conditions. The photometry was cleaned by applying a $3 \sigma$ threshold to remove outliers. In the vicinity of TWA 23 , we selected two stars TYC 72352841 ( $\mathrm{RA}=12: 07: 31.6 ; \mathrm{DEC}=-32: 49: 33.4 ; \mathrm{V}=11.04 \mathrm{mag})$ and 2MASS J12073387-3246529 (RA = 12:07:33.9; $\mathrm{DEC}=-32: 46: 52.9 ; \Delta \mathrm{R}=2.5$ mag) which did not vary during the whole run. Therefore, they turned out to be suitable to serve as comparison (C) and check (CK) stars, respectively, for our differential photometry. During the observation run, the differential magnitude of the check minus the comparison star remained constant with a mean value of $\Delta \mathrm{R}=2.50 \mathrm{mag}$ and a standard deviation $\sigma=0.020 \mathrm{mag}$.

The Generalized-Lomb-Scargle (GLS) periodogram (bottom-left panel of Fig. 4) shows a number of highly-significant power peaks. In order of decreasing power we have the following three periods: $\mathrm{P}=5.54 \pm 0.01 \mathrm{~d}, \mathrm{P}=1.2 \pm 0.005 \mathrm{~d}$ and $\mathrm{P}=$ $2.52 \pm 0.008 \mathrm{~d}$. In the CLEAN periodogram (bottom-right panel of Fig. 4) the dominant period is $\mathrm{P}=5.54 \mathrm{~d}$ and the secondary period is $\mathrm{P}=2.52 \mathrm{~d}$. The period at $\mathrm{P}=$ $1.2 \mathrm{~d}$ disappeared since it is the beat period of $\mathrm{P}=5.54 \mathrm{~d}$ and arising from the data sampling spectral window. Assuming $\mathrm{P}=5.54 \mathrm{~d}$ to be real and filtering it out from the data, we re-computed the GLS periodogram and found the dominant period to be $\mathrm{P}=2.52 \mathrm{~d}$ (see Fig. 5). Therefore, the pre-whitening of the primary periodicity has given us the confirmation that a secondary periodicity was present and that was of stellar origin rather than an aliasing effect arising from the data sampling. 

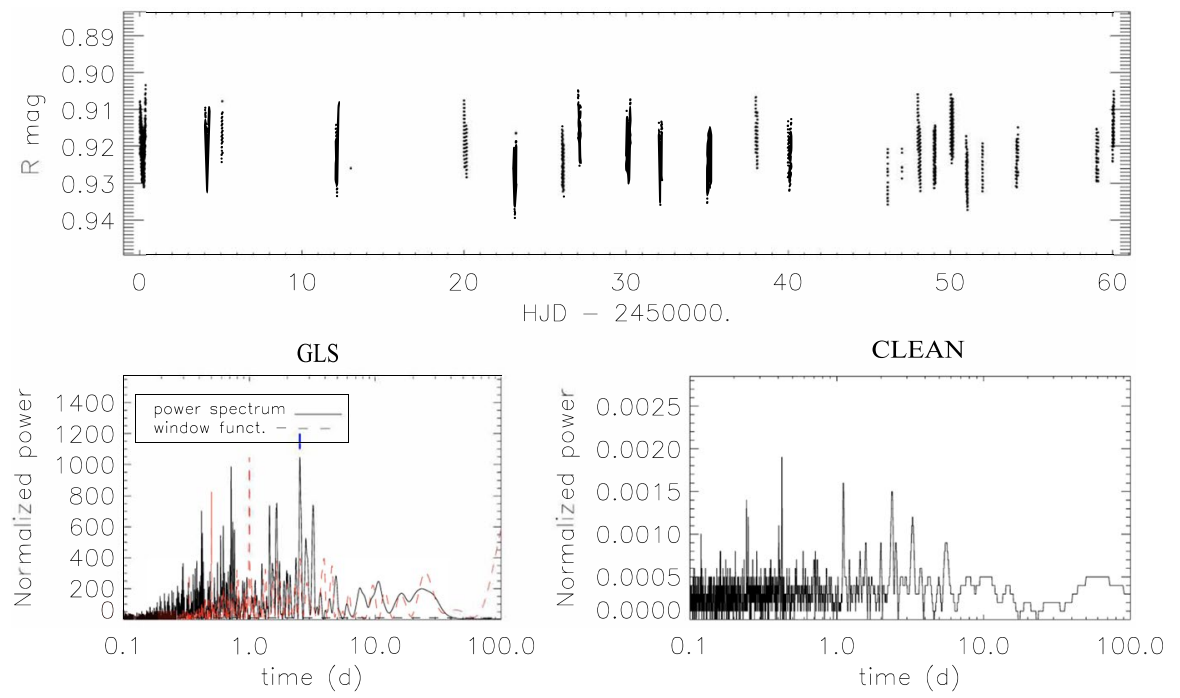

Fig. 5 Same as in Fig. 4, but after filtering out the primary period $\mathrm{P}=5.54 \mathrm{~d}$, with indication of the secondary periodicity at $\mathrm{P}=2.52 \mathrm{~d}$
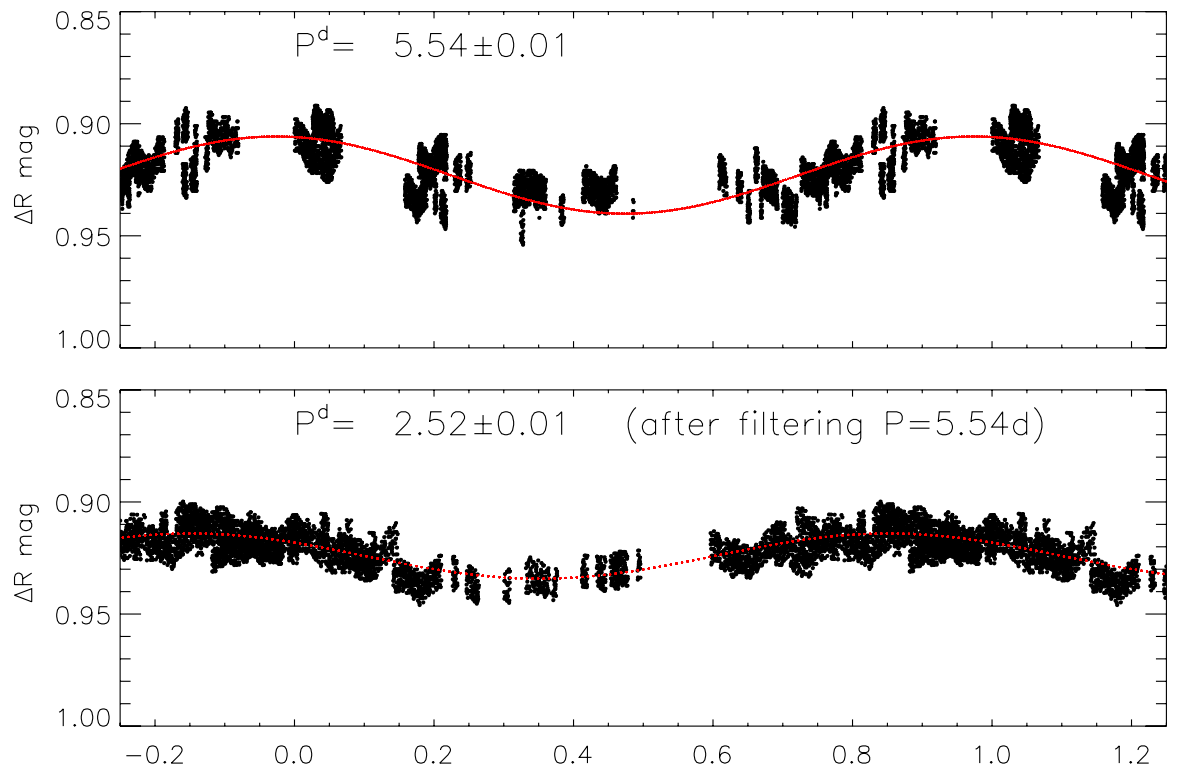

Fig. 6 Top panel: Differential R-band light curve of TWA 23 phased with the $\mathrm{P}=5.54$ d rotation period. The solid red line is the sinusoidal fit with ampliude ${ }^{\Delta} \mathrm{R}=0.034$ mag. Bottom panel: Differential R-band light curve of TWA 23 phased with the $\mathrm{P}=2.52 \mathrm{~d}$ rotation period, after pre-withening of the $\mathrm{P}=5.54 \mathrm{~d}$ period. The solid red line is the sinusoidal fit with amplitude $\Delta \mathrm{R}=0.020 \mathrm{mag}$. 
In Fig. 6, we show the KKO R-band light curves of TWA 23 which are phased with the primary period $\mathrm{P}=5.54 \mathrm{~d}$ and, after filtering, with the secondary period $\mathrm{P}$ $=2.52 \mathrm{~d}$.

Our finding of two rotation periods likely related to the two components of TWA 23 have been subsequently confirmed by the TESS photometry that was collected a few years later.

\subsubsection{TESS}

TESS observed TWA 23 in Sector 10 (from March 26 until April 22, 2019) with 2-minute cadence, providing us with the up-to-date highest-precision photometric time series of this star, $\sigma=0.001 \mathrm{mag}$ (see Table 1). To search for the rotation period in TESS data, we analysed the PDCSAP (Pre-search Data Conditioning Simple Aperture Photometry) fluxes using Autocorrelation Function (ACF), GLS, and CLEAN. Results are summarized in Fig. 7.

The autocorrelation function shows the first local maximum at $\mathrm{P}=5.29 \pm 0.54 \mathrm{~d}$ (second-left panel of Fig. 7), which we recognize to be the period of the flux modulation clearly visible in the TESS timeseries (top panel of Fig. 7). The ACF maximum at $\mathrm{P}=5.29 \mathrm{~d}$ is between two deep minima with no hint for a secondary periodicity. However, after filtering the 5.29-d periodicity from the data, the new ACF shows clear evidence of a secondary highly significant periodicity at $\mathrm{P}=$ $2.49 \pm 0.12 \mathrm{~d}$ (second-right panel of Fig. 7).

GLS finds a highly significant period at $\mathrm{P}=5.52 \pm 0.62 \mathrm{~d}$ (third-left panel of Fig. 7), which is in agreement within the respective uncertainties with the period inferred from the ACF. The primary power peak is accompanied by side-lobe like power peaks, which generally arise from the convolution of the Discrete Fourier Transform with the Spectral Window Function. However, after filtering the 5.52-d periodicity from the data, the new periodogram shows two highly significant power peaks at $\mathrm{P}=2.49 \pm 0.12 \mathrm{~d}$ and $\mathrm{P}=2.86 \pm 0.12 \mathrm{~d}$ (third-right panel of Fig. 7).

CLEAN finds a highly significant period at $\mathrm{P}=5.52 \pm 0.62 \mathrm{~d}$ (fourth-left panel of Fig. 7). Differently than ACF and GLS, the CLEAN does a pre-whitening of the dominant power peaks as integral part of its algorithm. Consequently, two other significant lower-power peaks at $\mathrm{P}=2.49 \pm 0.12 \mathrm{~d}$ and $\mathrm{P}=2.86 \pm 0.12 \mathrm{~d}$ are also visible in its periodogram. These secondary periods become even more clearly visible when, following the same procedure used with GLS, the primary period is filtered out (fourth-right panel of Fig. 7). The $\mathrm{P}=2.86 \pm 0.12 \mathrm{~d}$ found by GLS and CLEAN is at half of the primary period and likely arises from a spot group centered on the opposite hemisphere. Differently, the $\mathrm{P}=2.49 \mathrm{~d}$ period is unrelated to the primary period and likely originates from the variability of the undetected secondary component.

From this analysis, it is evident that when using either ACF or GLS to detect periodicities, it should be advisable to re-run the methods after filtering out the primary period measured in the original series. Such a practice should prevent us from missing valuable information which, although present in the time series may be "masked" by the dominancy of the primary peak. 

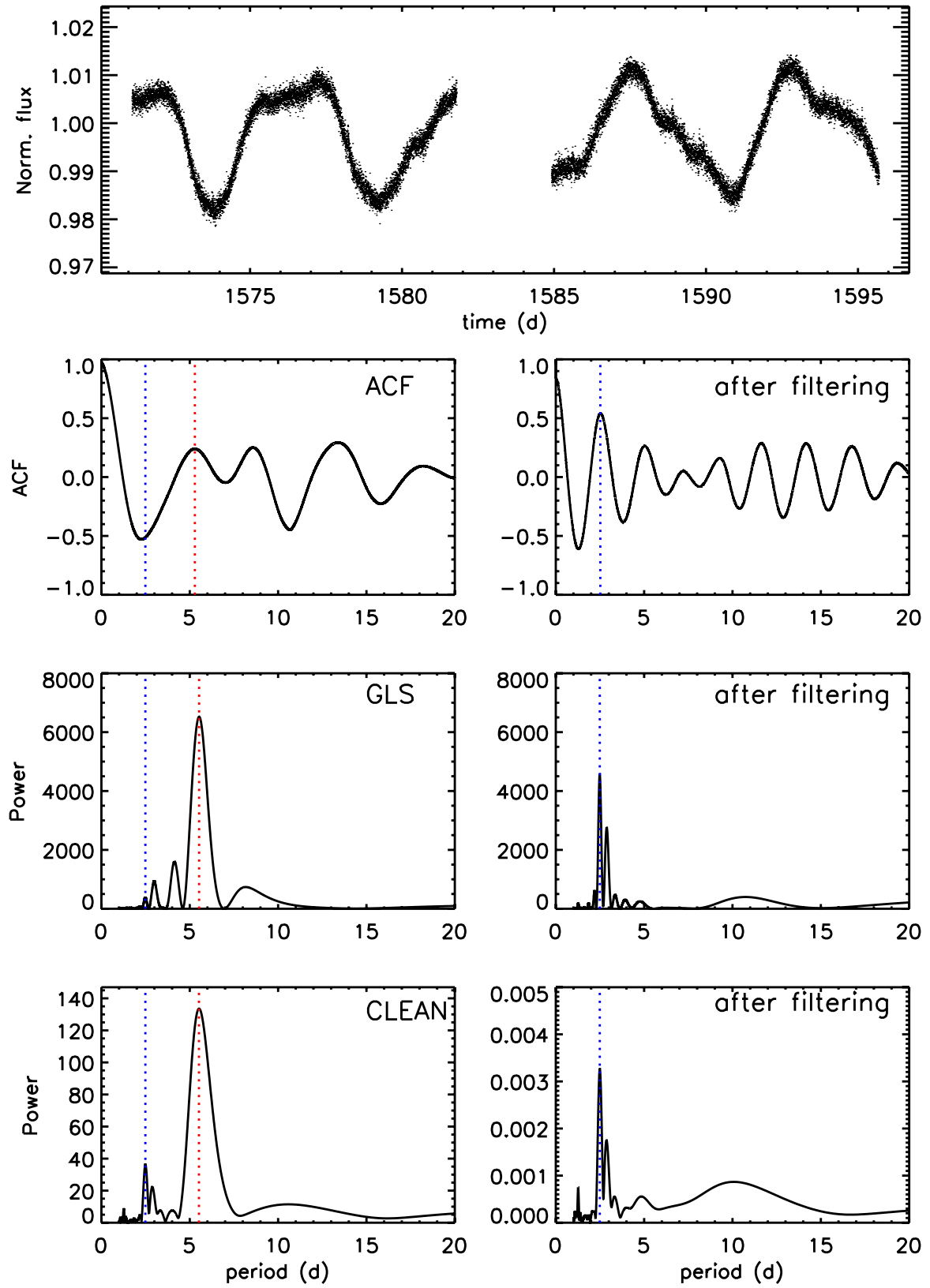

Fig. 7 Summary of TESS photometry of TWA 23. The left panels show the ACF, the GLS and CLEAN periodograms of original data, whereas the right panels show the corresponding results after filtering the primary $\mathrm{P}=5.52-\mathrm{d}$ periodicity. Vertical dotted lines indicate the position of the primary period (red) and secondary period (blue) 


\section{Discussion}

As mentioned in the Introduction, the analysis of Kepler/K2 time series of open clusters has shown an incidence of about $20 \%$ of multiperiodic stars, that is stars whose periodograms exhibit the presence of at least two distinct and highly significant power peaks. Whereas the multiperiodicity can arise from surface differential rotation for G- and K-type stars, for most M-type stars that arises from binarity. For example, [25] have analysed a sample of 129 among the brighter 259 multiperiodic stars discovered by [17] in the Upper Scorpius association. They found that more than $50 \%$ are indeed close binaries and the double periodicity represents the rotation periods of the two components. On one hand the periodogram analysis can in principle reveal the binary nature of unresolved close binaries, on the other hand it can be used to confirm the binary nature when suspected from spectroscopic investigation, and with higher confidence in the case of $M$ stars. In this framework, we have searched for a confirmation from photometric analysis of the binary nature of TWA 23.

Shkolnik et al. [20], Bailey et al. [2], and more recently [29] have provided clear spectroscopic evidence of the binary nature of TWA 23. Shkolnik et al. [20] assumed both components to be M3-type stars on the basis of a single-epoch spectrum. On the basis of 14 spectra (12 of which collected during the course of the same year), [2] concluded that one component is likely of later spectral type considering the small amplitude $\left(\sigma=2.42 \mathrm{~km} \mathrm{~s}^{-1}\right)$ of the RV variations.

Our analysis based on photometric time series confirms the binary nature of TWA 23, thanks to the detection of photometric variability from the spatially unresolved secondary component, and allowed us to measure its rotation period. Moreover, our analysis also suggests that the secondary component is indeed of later, and presumably M4, spectral type, supporting the conclusions of [2].

The rotation periods we measured for the $M$ components of the TWA23 system fit well the rotation period distribution of M-type members of young clusters. For instance, [11] show that in the Upper Scorpius association, which has an age comparable to that of the TWA association, the average rotation period decreases from about $7 \mathrm{~d}$ to $1 \mathrm{~d}$ in the color range $4<(V-K)_{0}<6.5 \mathrm{mag}$, roughly corresponding to spectral types from M0 to M6 (see Fig. 2 in [11]). Following this pattern of decreasing rotation period versus color among young $\mathrm{M}$ stars, the shorter rotation period $\mathrm{P}=2.52 \mathrm{~d}$ measured for the secondary component of TWA 23 can be ascribed to its later spectral type with respect to the primary component. For instance, a M3-type star in the Upper Scorpius association has an average rotation period of about 4 days whereas a M4-type star has a rotation period of about 2 days, that is half value. This ratio suggests that in our case, the secondary component of TWA 23, which has half rotation period with respect to the primary, differs by only one sub-class, thus it is a M4-type star.

In the TESS photometry we found the amplitude of variability of the M3 component to be three times larger than of the secondary component $(0.0118$ versus 0.0038 mag). Assuming that the secondary, owing to its faster rotation has a level of activity double with respect to the primary, taking into account the light dilution effect, 
being both components unresolved in the TESS photometry, we find that a flux variability of $5 \%$ and $10 \%$ of the primary M3-type and of the secondary M4-type, respectively, would generate the measured magnitude variations.

The knowledge of the rotation period, when combined with stellar radius and projected rotational velocity, allows to infer the inclination of the stellar rotation axis, providing additional information on the stellar properties. Assuming V = $12.55 \mathrm{mag}$ (from ASAS time series) as the unspotted magnitude, we correct for binarity the magnitudes of both components and considering the distance $d=55.7 \pm 0.3 \mathrm{pc}$, we derive the absolute magnitude $\left(\mathrm{M}_{\mathrm{V}}\right)_{A}=9.57 \mathrm{mag}$ and $\left(\mathrm{M}_{\mathrm{V}}\right)_{B}=10.57 \mathrm{mag}$. After applying the bolometric correction $\mathrm{BC}_{A}=-2.03 \mathrm{mag}$ and $\mathrm{BC}_{B}=-2.43 \mathrm{mag}$ [14], we derive the luminosity $\left(\mathrm{L} / \mathrm{L}_{\odot}\right)_{A}=0.077 \pm 0.009$ and $\left(\mathrm{L} / \mathrm{L}_{\odot}\right)_{B}=0.043 \pm 0.005$.

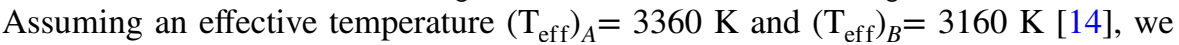
infer a stellar radius $\mathrm{R}_{A}=0.82 \pm 0.14 \mathrm{R}_{\odot}$ and $\mathrm{R}_{B}=0.69 \pm 0.12 \mathrm{R}_{\odot}$. The measured $v \sin =14.78 \mathrm{~km} \mathrm{~s}^{-1}$ is consistent only with radius and rotation period of the secondary M4 components, yielding an inclination $i=90 \pm 10^{\circ}$. The M3 component with a period $\mathrm{P}=5.54 \mathrm{~d}$ and a radius $\mathrm{R}=0.82 \mathrm{R}_{\odot}$ is expected to have $v \sin i \simeq 7.5 \mathrm{~km} \mathrm{~s}^{-1}$. It is likely that the spectra of both components were merged at the epoch of observations and the measured projected rotational velocity refers to the broader spectral lines of the faster component, which have overcome the narrower lines of the slower component.

\section{Conclusions}

We have carried out a photometric analysis of TWA 23, a young member of the 8-Myr old TWA association. Our analysis, based on archival and newly-collected photometry has allowed us to measure the rotation periods of both spatially-unresolved components of this star: $\mathrm{P}=5.52 \pm 0.6 \mathrm{~d}$ for the primary and $\mathrm{P}=2.49 \pm 0.12 \mathrm{~d}$ for the secondary component, confirming the suspicion based on spectroscopic observation that it was an SB2 system. Moreover, in agreement with spectroscopic evidence that the secondary has later spectral type, our analysis also suggests that this system consists of M3+M4 components.

Three different period search methods have been applied, the Autocorrelation Function, the Generalized Lomb-Scargle, and the CLEAN analyses. In fact, the most robust period determination generally comes when two or more methods are adopted and the results are a-posteriori combined.

We have presented in this paper this specific case to show that, even with the highest-quality photometry, like that collected by TESS, and the straitforward detection of the star's rotation period, which appears as dominant periodicity in both GLS and ACF periodograms, the practice of pre-whithening this primary period and to look for secondary perioditicies is strongly advised. In fact, the presence of a secondary periodicity in the TESS time series, which unveils the true binary nature of TWA 23, is detected with high significance only after the pre-whithening of the primary periodicity. Considering the large fraction of close binaries that, being undetected yet, are in the input catalogs of the ongoing and future space mission, like TESS and PLATO, respectively, it is important that in the automatic period search 
pipelines the pre-whithening be integral part, to avoid missing relevant information present in the data but not properly unveiled.

Acknowledgements Research on stellar activity at the INAF-Catania Astrophysical Observatory is supported by MUR (Ministero Universitá e Ricerca). The TESS data presented in this paper were obtained from the Mikulski Archive for Space Telescopes (MAST). STScI is operated by the Association of Universities for Research in Astronomy, Inc., under NASA contract NAS5-26555. Support for MAST for non-HST data is provided by the NASA Office of Space Science via grant NNX13AC07G and by other grants and contracts. This research has made use of the SIMBAD database, operated at CDS, Strasbourg, France. This paper makes use of data from the DR1 of the WASP data (Butters et al. 2010) as provided by the WASP consortium, and the computing and storage facilities at the CERIT Scientific Cloud, reg. no. CZ.1.05/3.2.00/08.0144 which is operated by Masaryk University, Czech Republic. The CSS survey is funded by the National Aeronautics and Space Administration under Grant No. NNG05GF22G issued through the Science Mission Directorate Near-Earth Objects Observations Program. The CRTS survey is supported by the U.S. National Science Foundation under grants AST-0909182 and AST-1313422. SM acknowledges financial support from Progetto Mainstream "Stellar evolution and asteroseismology in the context of the PLATO space mission" (PI: S. Cassisi).

Open Access This article is licensed under a Creative Commons Attribution 4.0 International License, which permits use, sharing, adaptation, distribution and reproduction in any medium or format, as long as you give appropriate credit to the original author(s) and the source, provide a link to the Creative Commons licence, and indicate if changes were made. The images or other third party material in this article are included in the article's Creative Commons licence, unless indicated otherwise in a credit line to the material. If material is not included in the article's Creative Commons licence and your intended use is not permitted by statutory regulation or exceeds the permitted use, you will need to obtain permission directly from the copyright holder. To view a copy of this licence, visit http://creativecommons.org/licen ses/by/4.0/.

\section{References}

1. Aigrain, S., Pont, F., Zucker, S.: A simple method to estimate radial velocity variations due to stellar activity using photometry. MNRAS 419(4), 3147-3158 (2012). https://doi.org/10.1111/j.13652966.2011.19960.x. arxiv:1110.1034

2. Bailey, I., John, I., White, R.J., Blake, C.H., Charbonneau, D., Barman, T.S., Tanner, A.M., Torres, G.: Precise infrared radial velocities from Keck/NIRSPEC and the search for young planets. APJ 749(1), 16 (2012). https://doi.org/10.1088/0004-637X/749/1/16. arxiv:1202.0300

3. Barnes, S.A.: On the rotational evolution of solar- and late-type stars, its magnetic origins, and the possibility of stellar gyrochronology. APJ 586(1), 464-479 (2003). https://doi.org/10.1086/367639. arxiv:astro-ph/0303631

4. Box, G.E.P., Jenkins, G.M.: Time series analysis. Forecasting and control. In: Holden-Day Series in Time Series Analysis (1976)

5. de la Reza, R., Pinzón, G.: On the rotation of post-t tauri stars in associations. AJ 128, 1812-1824 . https://doi.org/10.1086/423439. arxiv:astro-ph/0406412

6. Drake, A.J., Djorgovski, S.G., Mahabal, A., Beshore, E., Larson, S., Graham, M.J., Williams, R., Christensen, E., Catelan, M., Boattini, A., Gibbs, A., Hill, R., Kowalski, R.: First results from the catalina real-time transient survey. APJ 696, 870-884 (2009). https://doi.org/10.1088/0004-637X/ 696/1/870. arxiv:0809.1394

7. Durkan, S., Janson, M., Ciceri, S., Brandner, W., Schlieder, J., Henning, J., Bonnefoy, M., Kankare, J., Watson, C.A.: A Radial velocity survey of spatially resolved young, low-mass binaries. AAP 618, A5 (2018). https://doi.org/10.1051/0004-6361/201732156. arxiv:1806.05133

8. Gagné, J., Mamajek, E.E., Malo, L., Riedel, A., Rodriguez, D., Lafrenière, D., Faherty, J.K., RoyLoubier, O., Pueyo, L., Robin, A.C., Doyon, R.: BANYAN. XI. The BANYAN $\Sigma$ multivariate bayesian algorithm to identify members of young associations with 150 pc. APJ 856(1), 23 (2018). https://doi.org/10.3847/1538-4357/aaae09. arxiv:1801.09051 
9. Jayawardhana, R., Coffey, J., Scholz, A., Brandeker, A., van Kerkwijk, M.H.: Accretion disks around young stars: lifetimes, disk locking, and variability. APJ 648, 1206-1218 (2006). https://doi. org/10.1086/506171. arxiv:astro-ph/0605601

10. Mentuch, E., Brandeker, A., van Kerkwijk, M.H., Jayawardhana, R., Hauschildt, P.H.: Lithium depletion of nearby young stellar associations. APJ 689, 1127-1140 (2008). https://doi.org/10.1086/ 592764. arxiv:0808.3584

11. Messina, S.: Evidence from stellar rotation for early disc dispersal owing to close companions. AAP 627, A97 (2019). https://doi.org/10.1051/0004-6361/201834681. arxiv:0808.3584

12. Messina, S., Desidera, S., Lanzafame, A.C., Turatto, M., Guinan, E.F.: RACE-OC project: rotation and variability in the $\epsilon$ chamaeleontis, octans, and argus stellar associations. AAP 532, A10 (2011). https://doi.org/10.1051/0004-6361/201016116. arxiv:1104.2986

13. Parker, E.N.: Book-review - cosmical magnetic fields - their origin and their activity. Astronomy Quarterly 3, 201 (1979)

14. Pecaut, M.J., Mamajek, E.E.: Intrinsic colors, temperatures, and bolometric corrections of pre-mainsequence stars. APJS 208, 9 (2013). https://doi.org/10.1088/0067-0049/208/1/9. arxiv:1307.2657

15. Pojmanski, G.: The All Sky Automated Survey. ACTAA 47, 467-481 (1997). arxiv:astro-ph/9712146

16. Pollacco, D., Skillen, I., Collier Cameron, A., Christian, D., Irwin, J., Lister, T., Street, R., West, R., Clarkson, W., Evans, N., Fitzsimmons, A., Haswell, C., Hellier, C., Hodgkin, S., Horne, K., Jones, B., Kane, S., Keenan, F., Norton, A., Osborne, J., Ryans, R., Wheatley, P.: The WASP project and superwasp camera. APSS 304(1-4), 253-255 (2006). https://doi.org/10.1007/s10509-006-9124-X

17. Rebull, L.M., Stauffer, J.R., Cody, A.M., Hillenbrand, L.A., David, T.J., Pinsonneault, M.: Rotation of low-mass stars in upper scorpius and $\rho$ ophiuchus with K2. AJ 155, 196 (2018). https://doi.org/ 10.3847/1538-3881/aab605. arxiv:1803.04440

18. Roberts, D.H., Lehar, J., Dreher, J.W.: Time series analysis with clean - part one - derivation of a spectrum. AJ 93, 968 (1987). https://doi.org/10.1086/114383

19. Santos, A.R.G., García, R.A., Mathur, S., Bugnet, L., van Saders, J.L., Metcalfe, T.C., Simonian, G.V.A., Pinsonneault, M.H.: Surface rotation and photometric activity for kepler targets. I. M and K main-sequence stars. APJS 244(1), 21 (2019). https://doi.org/10.3847/1538-4365/ab3b56. arxiv: 1908.05222

20. Shkolnik, E.L., Liu, M.C., Reid, I.N., Dupuy, T., Weinberger, A.J.: Searching for Young M Dwarfs with GALEX. APJ 727(1), 6 (2011). https://doi.org/10.1088/0004-637X/727/1/6. arxiv:1011.2708

21. Skumanich, A.: Time Scales for Ca II Emission Decay, Rotational Braking, and Lithium Depletion. APJ 171, 565 (1972). https://doi.org/10.1086/151310

22. Song, I., Zuckerman, B., Bessell, M.S.: New Members of the TW Hydrae Association, $\beta$ Pictoris Moving Group, and Tucana/Horologium Association. APJ 599, 342-350 (2003). https://doi.org/10. $1086 / 379194$

23. Spada, F., Lanzafame, A.C., Lanza, A.F., Messina, S., Collier Cameron, A.: Modelling the rotational evolution of solar-like stars: the rotational coupling time-scale. MNRAS 416, 447-456 (2011). https://doi.org/10.1111/j.1365-2966.2011.19052.x. arxiv:1105.3125

24. Stellingwerf, R.F.: Period determination using phase dispersion minimization. APJ 224, 953-960 (1978). https://doi.org/10.1086/156444

25. Tokovinin, A., Briceño, C.: Yes, multi-periodic dwarfs in upper scorpius are binaries. AJ 156(3), 138 (2018). https://doi.org/10.3847/1538-3881/aad906. arxiv: 1808.02544

26. Torrence, C., Compo, G.P.: A practical guide to wavelet analysis. Bulletin of the American Meteorological Society 79(1), 61-78 (1998). https://doi.org/10.1175/1520-0477(1998)079<0061:APGTW $\mathrm{A}>2.0 . \mathrm{CO} ; 2$

27. Weiss, N.O.: Solar and stellar dynamos. In: Proctor, M.R.E., Gilbert, A.D. (eds) Lectures on Solar and Planetary Dynamos, p 59

28. Zechmeister, M., Kürster, M.: The generalised Lomb-Scargle periodogram. A new formalism for the floating-mean and Keplerian periodograms. AAP 496(2), 577-584 (2009). https://doi.org/10.1051/ 0004-6361:200811296. arxiv:0901.2573

29. Zuniga-Fernandez, S., Bayo, A., Elliott, P., Zamora, C., Corvalán, G., Haubois, X., Corral-Santana, J.M., Olofsson, J., Huélamo, N., Sterzik, M.F., Torres, C.A.O., Quast, G.R., Melo, C.H.F.: Search for associations containing young stars (SACY). VIII. An updated census of spectroscopic binary systems exhibiting hints of non-universal multiplicity among their associations. AAP 645, A30 (2021). https://doi.org/10.1051/0004-6361/202037830. arxiv:2010.08575 